\title{
Successive Approximation Method for Solving Wu-Zhang Systems of (1 + 1) Dimensional
}

\author{
Abdulghafor M. Al-Rozbayani*, Abdulbaset H. Shammar \\ Department of Mathematics, College of Computer Sciences and Mathematics, University of Mosul, Mosul, Iraq \\ Email:^abdulghafor_rozbayani@uomosul.edu.iq, abdulbasithamid@uomosul.edu.iq
}

How to cite this paper: Al-Rozbayani, A.M. and Shammar, A.H. (2020) Successive Approximation Method for Solving WuZhang Systems of $(1+1)$ Dimensional. Open Access Library Journal, 7: e6601. https://doi.org/10.4236/oalib.1106601

Received: July 11, 2020

Accepted: August 15, 2020

Published: August 18, 2020

Copyright $\odot 2020$ by author(s) and Open Access Library Inc.

This work is licensed under the Creative Commons Attribution International License (CC BY 4.0).

http://creativecommons.org/licenses/by/4.0/

\begin{abstract}
In this section the Successive approximate method (S.A.M) introduced for solving the $\mathrm{Wu}$-Zhang systems, a $(1+1)$-dimensional nonlinear dispersive wave equation, this method shows us that the technique provided without disorder, in this model of convergence power series with a simple calculated ingredients and gives effective results.
\end{abstract}

\section{Subject Areas}

Numerical Analysis, Partial Differential Equations

\section{Keywords}

Successive Approximate Method, Wu-Zhang Systems

\section{Introduction}

The Nonlinear partial differential equations (NPDEs) have a big advantage in various fields of science like mathematical, physics, chemistry, biology, mechanics, and engineering. It is important to get a reliable solution and numerical solution for nonlinear systems. In this section we solved the Wu-Zhang Systems of one dimensional with initial condition, the $\mathrm{Wu}$-Zhang system can be written as

$$
\left\{\begin{array}{l}
u_{t}+u u_{x}+v_{x}=0 \\
v_{t}+v u_{x}+u v_{x}+\frac{1}{3} u_{x x x}=0
\end{array}\right.
$$

where $(v)$ is height water, $(u)$ is the surface speed of the water along $X$-direction (Mix, S. K. [1], Laing, Y. C.; Feng [2], Ja, Anwar; Jameel [3], Manaa, Saad A.; Easif [4], R. K., Saeed [5], Zheng, Xuedong; Chen [6], Manaa, Saad A.; Easif [4] [7], Hosseini, K.; Ansari [8], Khater, Mostafa M. A.; Attia [9], Jafari, M. A.; Aminataei, A. [10]). 


\section{Successive Approximation Method (S.A.M) [5]}

The successive approximation method from important and active methods to solve partial differential equations, and also is good method for solving any initial value problem

$$
\frac{\partial u}{\partial t}=f(u, t), u\left(x, t_{0}\right)=u_{0}
$$

It starts by observing that any solution to (2) must also be a solution to

$$
u(x, t)=u_{0}+\int_{t_{0}}^{t} f(u(x, s), s) \mathrm{d} s,
$$

Thus iterative these steps of solutions, we obtain on solution closer to the accurate solutions of (3), the S.A.M depends on the integral equation (3), as in the following:

$$
\begin{gathered}
u_{0}(x, t)=u_{0}, \\
u_{1}(x, t)=u_{0}+\int_{t_{0}}^{t} f\left(u_{0}(x, s), s\right) \mathrm{d} s, \\
u_{2}(x, t)=u_{0}+\int_{t_{0}}^{t} f\left(u_{1}(x, s), s\right) \mathrm{d} s, \\
u_{3}(x, t)=u_{0}+\int_{t_{0}}^{t} f\left(u_{2}(x, s), s\right) \mathrm{d} s,
\end{gathered}
$$

This process can be continued to obtain the $n^{\text {th }}$ approximate

$$
u_{n}(x, t)=u_{0}+\int_{t_{0}}^{t} f\left(u_{n-1}(x, s), s\right) \mathrm{d} s, \quad n=1,2, \cdots
$$

Therefore determine if $u_{n}(x, t)$ closer the solution $u(x, t)$ as $n$ increases. This is done by proving the following:

The sequence $\left\{u_{n}(x, t)\right\}$ converges to a limit $u(x, t)$, that is:

$$
\lim _{n \rightarrow \infty} u_{n}(x, t)=u(x, t), t_{0} \leq x \leq t .
$$

The limiting function $u(x, t)$ is a solution of (3) on the interval $t_{0} \leq x \leq t$. The solution $u(x, t)$ of $(3)$ is unique. A proof of these results can be depended along the lines of the corresponding proof for ordinary differential equations (Coddington, 1995).

\section{Application (S.A.M) to solve of Equation (1)}

In this section, we solve the $\mathrm{Wu}$-Zhang systems of one dimensional

$$
\begin{gathered}
\frac{\partial u(x, s)}{\partial s}=-u(x, s) \frac{\partial u(x, s)}{\partial x}-\frac{\partial v(x, s)}{\partial x} \\
\frac{\partial v(x, s)}{\partial s}=-v(x, s) \frac{\partial u(x, s)}{\partial x}-u(x, s) \frac{\partial v(x, s)}{\partial x}-\frac{1}{3} \frac{\partial^{3} u(x, s)}{\partial x^{3}}
\end{gathered}
$$

With initial condition: 


$$
\begin{gathered}
u(x, 0)=\frac{1}{3 k_{1}}\left[b_{0}+\sqrt{18 k_{1}^{3}+b_{0}^{2}} \tanh \left(\frac{\sqrt{54 k_{1}^{3}+3 b_{0}^{2}}}{6 k_{1}}\left(x+k_{2}\right)\right)\right] \\
v(x, 0)=k_{1}+\frac{b_{0}}{3 k_{1}}\left[\frac{1}{3 k_{1}}\left[b_{0}+\sqrt{18 k_{1}^{2}+b_{0}^{2}} \tanh \left(\frac{\sqrt{54 k_{1}^{3}+3 b_{0}^{2}}}{6 k 1}\left(x+k_{2}\right)\right)\right]\right] \\
-\frac{1}{2}\left[\frac{1}{3 k_{1}}\left[b_{0}+\sqrt{18 k_{1}^{3}+b_{0}^{2}} \tanh \left(\frac{\sqrt{54 k_{1}^{3}+3 b_{0}^{2}}}{6 k_{1}}\left(x+k_{2}\right)\right)\right]\right]^{2}
\end{gathered}
$$

where $b_{0}, k_{1}$ and $k_{2}$ are arbitrary constants.

By using S.A.M as follows:

Integrating both sides of Equations (4), (5) with respect to $(s)$, from $(0)$ to $(t)$, we get

$$
\begin{gathered}
u(x, s)=u(x, 0)-\int_{0}^{t}\left(u(x, s) \frac{\partial u(x, s)}{\partial x}+\frac{\partial v(x, s)}{\partial x}\right) \mathrm{d} s \\
v(x, s)=v(x, 0)-\int_{0}^{t}\left(v(x, s) \frac{\partial u(x, s)}{\partial x}+u(x, s) \frac{\partial v(x, s)}{\partial x}+\frac{1}{3} \frac{\partial^{3} u(x, s)}{\partial x^{3}}\right) \mathrm{d} s
\end{gathered}
$$

Using the initial condition in Equations (8), (9) we obtain:

$$
\begin{gathered}
u(x, s)=u_{0}-\int_{0}^{t}\left(u(x, s) \frac{\partial u(x, s)}{\partial x}+\frac{\partial v(x, s)}{\partial x}\right) \mathrm{d} s \\
v(x, s)=v_{0}-\int_{0}^{t}\left(v(x, s) \frac{\partial u(x, s)}{\partial x}+u(x, s) \frac{\partial v(x, s)}{\partial x}+\frac{1}{3} \frac{\partial^{3} u(x, s)}{\partial x^{3}}\right) \mathrm{d} s
\end{gathered}
$$

We substituting initial conditions $u(x, 0)$ and $v(x, 0)$ in the integral Equations (10), (11) to get a first approximation $u_{1}(x, t)$ and $v_{1}(x, t)$

$$
\begin{gathered}
u_{1}(x, s)=u_{0}-\int_{0}^{t}\left(u_{0}(x, s) \frac{\partial u_{0}(x, s)}{\partial x}+\frac{\partial v_{0}(x, s)}{\partial x}\right) \mathrm{d} s \\
v_{1}(x, s)=v_{0}-\int_{0}^{t}\left(v_{0}(x, s) \frac{\partial u_{0}(x, s)}{\partial x}+u_{0}(x, s) \frac{\partial v_{0}(x, s)}{\partial x}+\frac{1}{3} \frac{\partial^{3} u_{0}(x, s)}{\partial x^{3}}\right) \mathrm{d} s
\end{gathered}
$$

Then this $u_{1}(x, t)$ and $v_{1}(x, t)$ is substituted again in the integral of (10), (11) after replacing $(t)$ by $(s)$ to get a second approximation $u_{2}(x, t)$ and $v_{2}(x, t)$

$$
\begin{gathered}
u_{2}(x, s)=u_{0}-\int_{0}^{t}\left(u_{1}(x, s) \frac{\partial u_{1}(x, s)}{\partial x}+\frac{\partial v_{1}(x, s)}{\partial x}\right) \mathrm{d} s \\
v_{2}(x, s)=v_{0}-\int_{0}^{t}\left(v_{1}(x, s) \frac{\partial u_{1}(x, s)}{\partial x}+u_{1}(x, s) \frac{\partial v_{1}(x, s)}{\partial x}+\frac{1}{3} \frac{\partial^{3} u_{1}(x, s)}{\partial x^{3}}\right) \mathrm{d} s
\end{gathered}
$$

This process can continued to get the $n^{\text {th }}$ approximation

$$
u_{n}(x, s)=u_{0}-\int_{0}^{t}\left(u_{n-1}(x, s) \frac{\partial u_{n-1}(x, s)}{\partial x}+\frac{\partial v_{n-1}(x, s)}{\partial x}\right) \mathrm{d} s
$$


$v_{n}(x, s)=v_{0}-\int_{0}^{t}\left(v_{n-1}(x, s) \frac{\partial u_{n-1}(x, s)}{\partial x}+u_{n-1}(x, s) \frac{\partial v_{n-1}(x, s)}{\partial x}+\frac{1}{3} \frac{\partial^{3} u(x, s)}{\partial x^{3}}\right) \mathrm{d} s$

For $n=1,2, \cdots$ to solve Equations (16), (17), we use the initial conditions $u(x, 0)$ and $v(x, 0)$ which are given in Equations (6) and (7), respectively. by using iterative steps for Equations (16), (17) we can obtain $u_{1}(x, t), u_{2}(x, t)$, $u_{3}(x, t)$ and $v_{1}(x, t), v_{2}(x, t), \quad v_{3}(x, t)$, after then substituting these values in the Equations (18) and (19)

$$
\begin{aligned}
& u=\sum_{n=0}^{\infty} u_{n}(x, t) \\
& v=\sum_{n=0}^{\infty} v_{n}(x, t)
\end{aligned}
$$

We get the following series:

$$
\begin{gathered}
\tilde{u}_{3}(x, t)=\frac{1}{3 k_{1}} b_{0}+\sqrt{18 k_{1}^{3}+b_{0}^{2}} \tanh \left(\frac{1}{6 k_{1}} \sqrt{54 k_{1}^{3}+3 b_{0}^{2}}\left(x+k_{2}\right)\right) \\
-\frac{1}{54} \frac{b_{0}\left(18 k_{1}^{3}+b_{0}^{2}\right) \sqrt{3} t}{k_{1}^{3} \cosh \left(\frac{1}{6 k_{1}} \sqrt{3} \sqrt{18 k_{1}^{3}+b_{0}^{2}}\left(x+k_{2}\right)\right)^{2}}+\cdots \\
\vdots \\
\tilde{v}_{3}(x, t)=k_{1}+\frac{1}{9 k_{1}^{2}} b_{0}\left(b_{0}+\sqrt{18 k_{1}^{3}+b_{0}^{2}} \tanh \left(\frac{1}{6 k_{1}} \sqrt{54 k_{1}^{3}+3 b_{0}^{2}}\left(x+k_{2}\right)\right)\right) \\
-\frac{1}{18 k_{1}^{2}}\left(b_{0}+\sqrt{18 k_{1}^{3}+b_{0}^{2}} \tanh \left(\frac{1}{6 k_{1}} \sqrt{54 k_{1}^{3}+3 b_{0}^{2}}\left(x+k_{2}\right)\right)\right)^{2} \\
+\frac{1}{162} \frac{\sinh \left(\frac{1}{6 k_{1}} \sqrt{54 k_{1}^{3}+3 b_{0}^{2}}\left(x+k_{2}\right)\left(18 k_{1}^{3}+b_{0}^{2}\right) \sqrt{54 k_{1}^{3}+3 b_{0}^{2}} b_{0} t\right)}{\cosh \left(\frac{1}{6 k_{1}} \sqrt{54 k_{1}^{3}+3 b_{0}^{2}}\left(x+k_{2}\right)^{3} k_{1}^{4}\right)}+\cdots
\end{gathered}
$$

Therefore, the exact solution of $\mathrm{Wu}$-Zhang equation is given by

$$
\begin{aligned}
& u(x, t)=\lim _{n \rightarrow \infty} \tilde{u}_{n}(x, t), \\
& v(x, t)=\lim _{n \rightarrow \infty} \tilde{v}_{n}(x, t),
\end{aligned}
$$

This solution is convergent to the exact solution

$$
\begin{aligned}
u(x, t) & =\frac{1}{3 k_{1}}\left[b_{0}+\sqrt{18 k_{1}^{3}+b_{0}^{2}} \tanh \left(\frac{\sqrt{54 k_{1}^{3}+3 b_{0}^{2}}}{6 k 1}\left(x-\frac{b_{0}}{3 k_{1}} t+k_{2}\right)\right)\right] \\
v(x, t)= & k_{1}+\frac{b_{0}}{3 k_{1}}\left[\frac{1}{3 k_{1}}\left[b_{0}+\sqrt{18 k_{1}^{3}+b_{0}^{2}} \tanh \left(\frac{\sqrt{54 k_{1}^{3}+3 b_{0}^{2}}}{6 k 1}\left(x-\frac{b_{0}}{3 k_{1}} t+k_{2}\right)\right)\right]\right] \\
& -\frac{1}{2}\left[\frac{1}{3 k_{1}}\left[b_{0}+\sqrt{18 k_{1}^{3}+b_{0}^{2}} \tanh \left(\frac{\sqrt{54 k_{1}^{3}+3 b_{0}^{2}}}{6 k_{1}}\left(x-\frac{b_{0}}{3 k_{1}} t+k_{2}\right)\right)\right]\right]^{2}
\end{aligned}
$$


The approximate results for $u(x, t)$ and $v(x, t)$ were compared with exact solution as showing in Table 1 and Table 2. Also we compare figure approximate solution with figure exact solution as showing in Figure 1 and Figure 2. Figure 3, error between approximate solution and exact solution.

In Figure 1 and Figure 2 the final results obtained from (S.A.M) were compared with the results of the exact solution when $b_{0}=0.1, k_{1}=0.05, k_{2}=0.1$, $t=0.2$. The comparison shows a good agreement between the results.

Table 1. Comparing exact solutions with approximate solutions (S.A.M) of the Wu-Zhang systems with initial conditions Equations (6), (7) where $b_{0}=0.1, k_{1}=0.05, k_{2}=0.1$, $t=0.2$ for $u(x, t)$.

\begin{tabular}{cccc}
\hline$X$ & $u_{\mathrm{SAM}}$ & $u_{\text {exact }}$ & $\left|u_{\mathrm{SAM}}-u_{\text {exact }}\right|$ \\
\hline-50 & -0.07119812060 & -0.07119812060 & $5.089992619 \times 10^{-29}$ \\
-40 & -0.07119812060 & -0.07119812060 & $1.807525608 \times 10^{-23}$ \\
-30 & -0.07119812060 & -0.07119812060 & $6.418769275 \times 10^{-18}$ \\
-20 & -0.07119812060 & -0.07119812060 & $2.279392226 \times 10^{-12}$ \\
-10 & -0.07119413194 & -0.07119413830 & $6.362404900 \times 10^{-9}$ \\
0 & 0.6478908251 & 0.6509522859 & $3061460790 \times 10^{-3}$ \\
10 & 1.404527229 & 1.404527118 & $1.113311969 \times 10^{-7}$ \\
20 & 1.404531454 & 1.404531454 & $1.765272343 \times 10^{-12}$ \\
30 & 1.404531454 & 1.404531454 & $4.971007506 \times 10^{-18}$ \\
40 & 1.404531454 & 1.404531454 & $1.399835854 \times 10^{-23}$ \\
50 & 1.404531454 & 1.404531454 & $3.941938236 \times 10^{-29}$ \\
\hline
\end{tabular}

Table 2. Comparing exact solutions with approximate solutions (S.A.M) of the Wu-Zhang systems with initial conditions Equations (6), (7) where $b_{0}=0.1, k_{1}=0.05, k_{1}=0.05$, $t=0.2$ for $v(x, t)$.

\begin{tabular}{cccc}
\hline$X$ & $v_{\text {SAM }}$ & $v_{\text {exact }}$ & $\left|v_{\text {SAM }}-v_{\text {exact }}\right|$ \\
\hline-50 & $7.000000000 \times 10^{-12}$ & $7.000000000 \times 10^{-12}$ & $7.000000000 \times 10^{-12}$ \\
-40 & $7.000000000 \times 10^{-12}$ & $7.000000000 \times 10^{-12}$ & $7.000000000 \times 10^{-12}$ \\
-30 & $6.999999972 \times 10^{-12}$ & $7.000000000 \times 10^{-12}$ & $7.000000028 \times 10^{-12}$ \\
-20 & $6.990087894 \times 10^{-12}$ & $7.000000000 \times 10^{-12}$ & $7.009912106 \times 10^{-12}$ \\
-10 & $2943074716 \times 10^{-6}$ & $2938409000 \times 10^{-6}$ & $4.671715510 \times 10^{-9}$ \\
0 & 0.2749015323 & 0.2720987513 & $2802780979 \times 10^{-3}$ \\
10 & $3117925519 \times 10^{-6}$ & $3200100000 \times 10^{-6}$ & $8.207448126 \times 10^{-8}$ \\
20 & $7.676417228 \times 10^{-5}$ & 0.0000000000000 & $7.676417228 \times 10^{-15}$ \\
30 & $2.161679351 \times 10^{-20}$ & 0.0000000000000 & $2.161679351 \times 10^{-20}$ \\
40 & $6.087289664 \times 10^{-26}$ & 0.0000000000000 & $6.087289664 \times 10^{-26}$ \\
50 & $1.714180972 \times 10^{-31}$ & 0.0000000000000 & $1.714180972 \times 10^{-31}$ \\
\hline
\end{tabular}



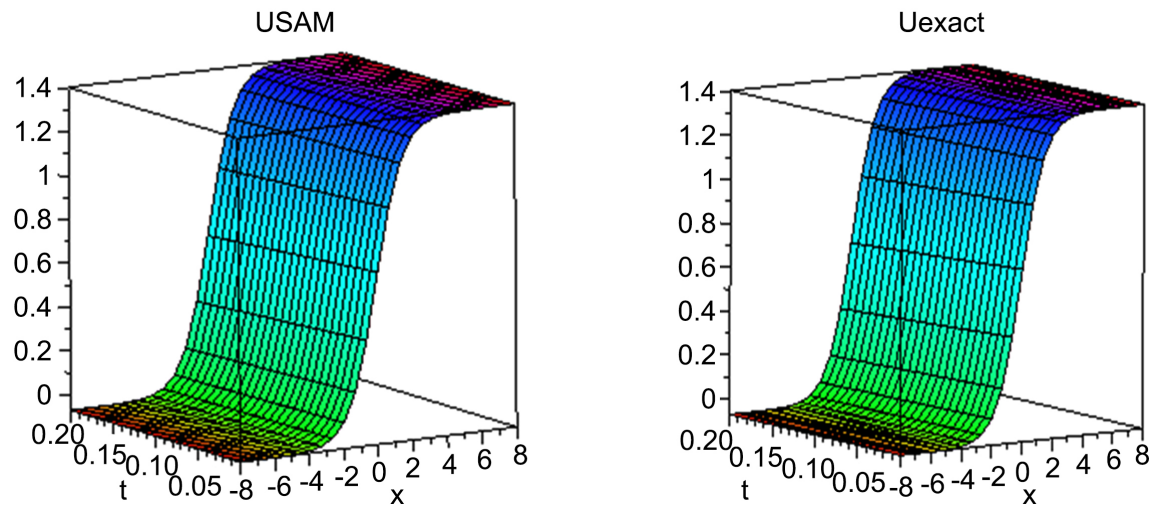

Figure 1. Showing matching approximate solution with exact solution. (USAM) approximate solution, (Uexact) exact solution.
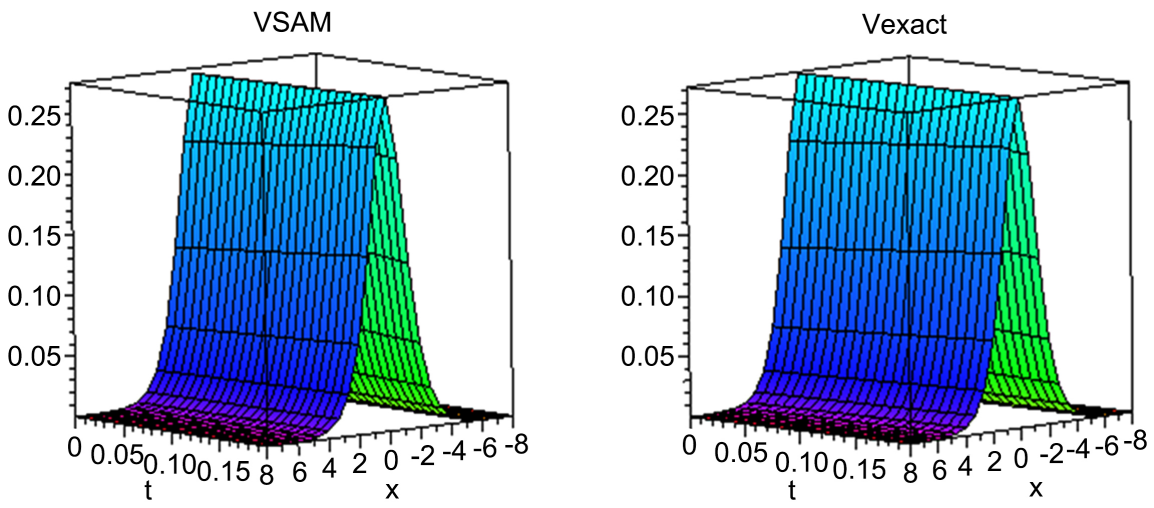

Figure 2. Showing matching approximate solution with exact solution. (VSAM) approximatesolution, (Vexact) exact solution.
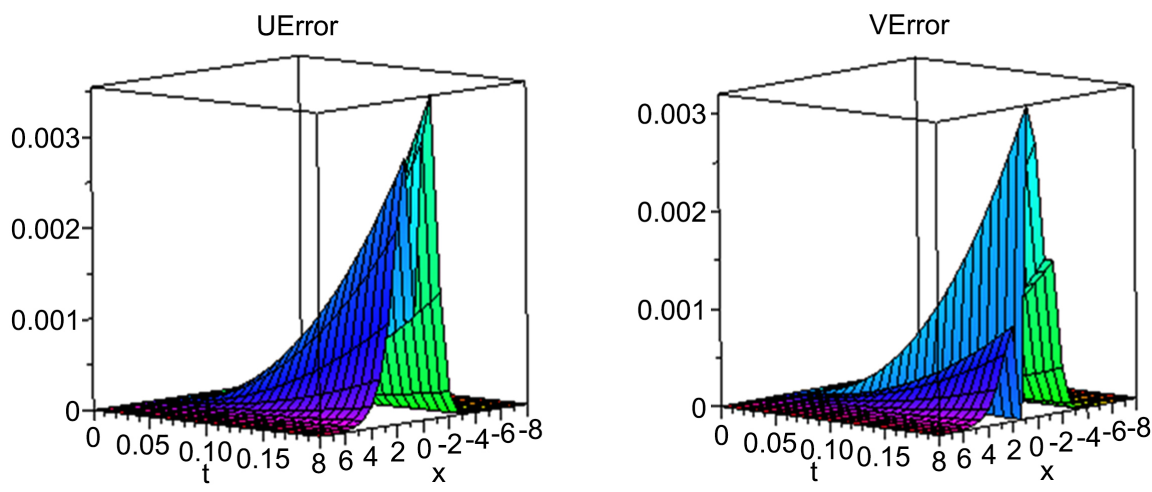

Figure 3. (UError) Showing error of the approximate solution for $u(x, t)$ with exact solution, (VError) Showing error of the approximate solution for $v(x, t)$ with exact solution.

\section{Conclusion}

In this search, the Successive Approximate Method (S.A.M) was used to get the approximate solution of $\mathrm{Wu}$-Zhang Systems. The results we got from this method are high efficient with big accurate and give a good convergence to the exact solution. 


\section{Acknowledgements}

The authors are very grateful to the University of Mosul/College of Computer Sciences and Mathematics for their provided facilities, which helped to improve the quality of this work.

\section{Conflicts of Interest}

The authors declare no conflicts of interest regarding the publication of this paper.

\section{References}

[1] Mix, S.K. (1966) Successive Approximation Methods for the Solution of the Optimal Control Problems. Automatica, 3, 135-149. https://doi.org/10.1016/0005-1098(66)90009-4

[2] Liang, Y.C., Feng, D.P., Lee, H.P., Lim, S.P. and Lee, K.H. (2002) Successive Approximation Training Algorithm for Feedforward Neural Networks. Neurocomputing, 42, 311-322. https://doi.org/10.1016/S0925-2312(01)00576-8

[3] Ja, A., Jameel, F. and Al, I. (2019) Soliton Solutions of Wu-Zhang System of Evolution Equations. Numerical and Computational Methods in Science and Engineering an International Journal, 11, 1-11.

[4] Manaa, S.A., Easif, F.H. and Mahmood, B.A. (2013) Successive Approximation Method for Solving Nonlinear Diffusion Equation with Convection Term. IOSR Journal of Engineering, 3, 28-31. https://doi.org/10.9790/3021-031232831

[5] Rk, S. (2006) Computational Methods for Solving System of Linear Volterra and Integro-Differential Equations. Ph. D. Thesis, Salahaddin University/Erbil, Iraq.

[6] Zheng, X., Chen, Y. and Zhang, H. (2003) Generalized Extended Tanh-Function Method and Its Application to $(1+1)$-Dimensional Dispersive Long Wave Equation. Physics Letters A, 311, 145-157. https://doi.org/10.1016/S0375-9601(03)00451-1

[7] Manaa, S.A., Easif, F.H. and Ali, B.Y. (2017) Long Wave Equations. International Journal of Advanced and Applied Sciences, 4, 98-103.

[8] Hosseini, K., Ansari, R. and Gholamin, P. (2011) Exact Solutions of Some Nonlinear Systems of Partial Differential Equations by Using the First Integral Method. Journal of Mathematical Analysis and Applications, 387, 807-814.

https://doi.org/10.1016/j.jmaa.2011.09.044

[9] Khater, M.M.A., Attia, R.A.M. and Lu, D. (2019) Numerical Solutions of Nonlinear Fractional Wu-Zhang System for Water Surface versus Three Approximate Schemes. Journal of Ocean Engineering and Science, 4, 144-148. https://doi.org/10.1016/j.joes.2019.03.002

[10] Jafari, M.A. and Aminataei, A. (2012) Method of Successive Approximations for Solving the Multi-Pantograph Delay Equations. Gen. Math. Notes, 8, 23-28. 